\title{
Regularization of fluctuations near the sonic horizon due to the quantum potential and its influence on the Hawking radiation
}

\author{
V. Fleurov ${ }^{1,3}$, R. Schilling ${ }^{2}$ \\ 1 Raymond and Beverly Sackler Faculty of Exact Sciences, \\ School of Physics and Astronomy, \\ Tel-Aviv University, Tel-Aviv 69978, Israel \\ 2 Johannes Gutenberg University, Mainz, Germany \\ 3 Max-Planck-Institut für Physik Komplexer Systeme, \\ Nöthnitzer Strasse 38, 01187 Dresden, Germany
}

\begin{abstract}
We consider dynamics of fluctuations in transonically accelerating Bose-Einstein condensates and/or luminous fluids (coherent light propagating in a Kerr nonlinear medium) using the hydrodynamic approach. It is known that neglecting the quantum potential (QP) leads to a singular behavior of quantum and classical fluctuations in the vicinity of the Mach (sonic) horizon, which in turn gives rise to the Hawking radiation. The neglect of QP is well founded at not too small distances $|x| \gg l_{h}$ from the horizon, where $l_{h}$ is the healing length. Taking the QP into account we show that a second characteristic length $l_{r}>l_{h}$ exists, such that the linear fluctuation modes become regularized for $|x| \ll l_{r}$. At $|x| \gg l_{r}$ the modes keep their singular behavior, which however is influenced by the QP. As a result we find a deviation of the high frequency tail of the spectrum of Hawking radiation from Planck's black body radiation distribution, which can be described by an effective Hawking temperature decreasing with increasing frequency. Similar results hold for the wave propagation in Kerr nonlinear media where the lengths $l_{h}$ and $l_{r}$ exist due to the nonlinearity.
\end{abstract}

PACS numbers: 03.75.Kk, 42.65.Wi, 04.70.Dy 


\section{INTRODUCTION}

Hawking radiation is one of the most impressive phenomena at the intersection of general relativity and quantum field theory. The account of quantum nature of the physical vacuum in curved space led to the prediction that a black hole - an object defined classically as an object that even light cannot escape - in fact emits radiation, $, 2,2$ which is characterized by the Planck's distribution of the black body at a certain temperature. The derivation involves singular behavior for the eigenmodes of the proper field equation in the vicinity of the horizon, which can be normalized only by using assumptions on Planck scale physics that cannot be justified completely. For a general discussion the reader may consult Ref. $[$.

Soon after that development it was suggested ${ }^{\underline{4}}$ to consider analogous phenomena in condensed matter physics where the "high-energy" (short-wavelength) physics is known. The suggestion was based on the observation that the derivation of the Hawking radiation uses only the linear wave equation in curved space-time, and does not make the actual use of the Einstein equations for gravity. The same conditions for wave propagation arise at the consideration of sound propagation in the fluid when the background flow is nontrivial. $\stackrel{4}{*}$ In particular, the background may be that of a stationary accelerating transonic flow where a surface appears, on which the fluid velocity equals that of sound, i.e where the Mach number is one, $M=1$. This surface has many features similar to that of the black hole horizon and is often called sonic or Mach horizon.

Currently theoretical studies of such artificial black holes became a very active field involving a variety of physical systems..$^{-12}$ Experimentally a white-hole horizon was observed in optical fibers, $\frac{13}{3}$ where the probe light was back-reflected from a moving soliton, and a black-hole horizon was observed in a Bose-Einstein condensate (BEC) system. $\underline{14}^{14}$ Continuing the study of optical fibers a radiation has been quite recently observed, ${ }^{15}$ which is a very promising contender to being analogous to Hawking radiation in a tabletop experiment. A "horizon physics" is currently intensively studied also in the surface water waves. $16-18$

Creating a stationary laminar transonic flow of an ordinary fluid, as required by the original proposal by Unruh, $\stackrel{4}{\underline{4}}$ may be a formidable task that is why many authors turn their attention to the BEC (see, e.g. Refs. 8-10), or as recently proposed, to a "luminous 


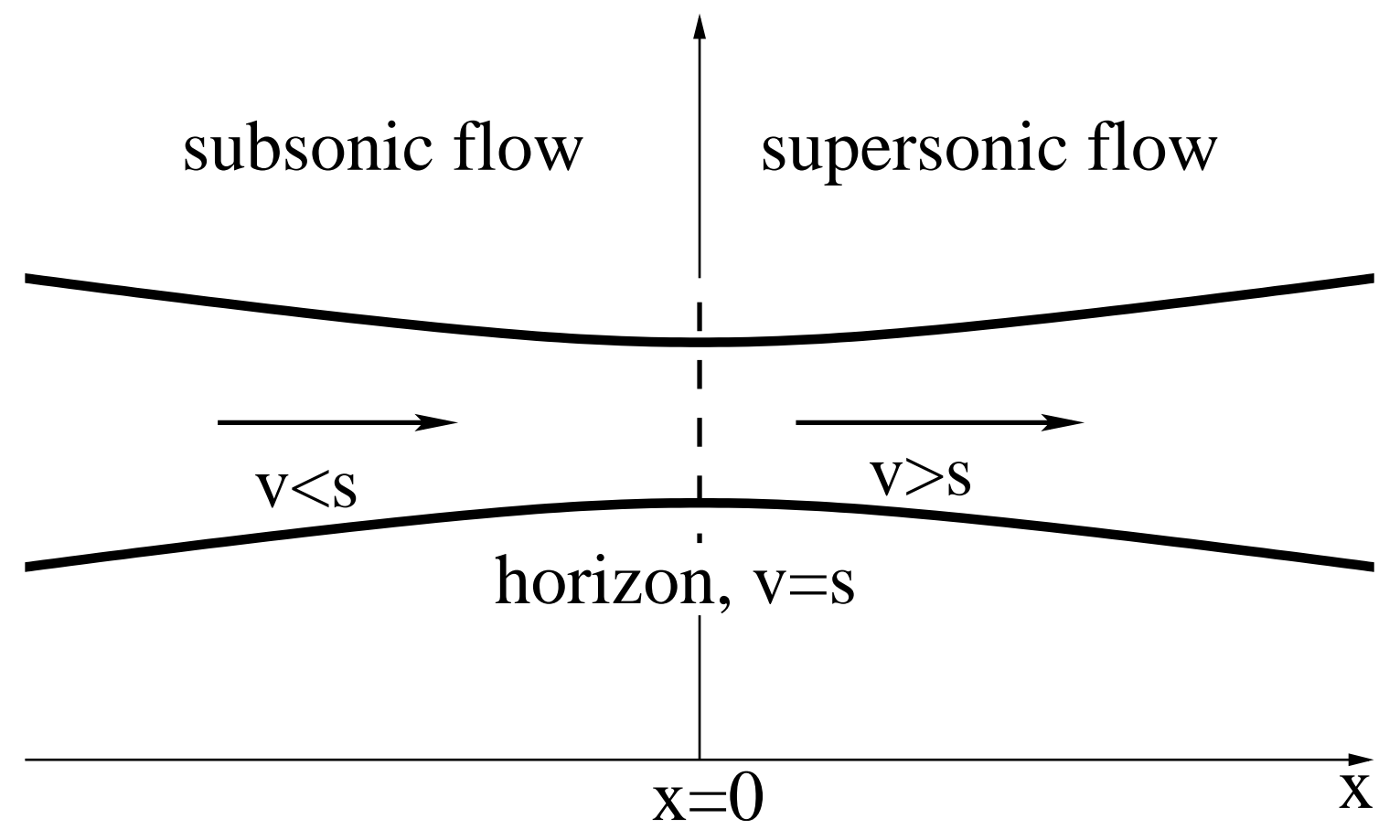

FIG. 1: A scheme of Laval nozzle as a vessel with non-monotonously varying cross-section. It is capable of accelerating a flow from subsonic to supersonic velocity. The sound velocity is reached in the throat (the narrowest cross-section) as shown by the dashed line where a Mach (sonic) horizon is formed.

fluid" 12 in an optical analog of the Laval nozzle. Generally the Laval nozzle (see, e.g., Refs. 19,20) schematically shown in Fig. 1 accelerates a flow from sub- to supersonic velocities. It uses the fact that the flow accelerates if the cross-section of the vessel decreases in the subsonic region, whereas in the supersonic region the cross-section must increase in order to accelerate the flow. An optical analog of the Laval nozzle $\frac{12}{2}$ is a properly shaped wave guide filled with a Kerr nonlinear medium, in which a coherent laser light propagates.

BEC is usually described by the Gross-Pitaevskii equation, which can be converted into two equivalent hydrodynamic equations by means of the Madelung transformation.21 A similar procedure exists also for the coherent light. 22 Analysis of the behavior of fluctuations near the Mach horizon based on these equations is usually carried out neglecting the role of the quantum potential (QP) $\stackrel{8,12}{12}$ This approximation can be well justified nearly everywhere except for the narrow vicinity of the sonic horizon due to the singular behavior of the fluctuations in this region. Neglecting QP the fluctuations are described by 
an equivalent Klein-Gordon equation for the phase fluctuations in a curved space with Schwarzschild metric, as first proposed in Ref. 4. The reader should also note that the fluctuation modes are then dispersionless, i.e. their frequency depends linearly on their wave-number, if QP is neglected.

At small distances from the horizon the contribution of the QP (kinetic energy operator for atoms in BEC or dispersion in luminous fluid) diverges and cannot be anymore neglected. This reminds the behavior of vacuum fluctuations near the black hole horizon on the Planck scale. Since in our case the physics on small scales is known the role of the QP in the near vicinity of the Mach horizon can and should be addressed.

Accounting for the QP introduces dispersion effects at higher frequencies corresponding to small length scales. The question how far such effects influence Hawking radiation has been studied during the last two decades. It seems that the first such study was done by Jacobson 23,24 . Whereas Ref. 23 had stated that a derivation of black hole radiation, taking the ultrashort distance physics into account, was missing, such a derivation was presented in Ref. 24. It has been concluded that boundary conditions can be formulated, which lead to the Hawking effect. In order to deduce quantitative results, e.g. for the flux and the spectrum, the mode equations based on specific dispersion laws were solved numerically $\underline{10}, 25-28$ and analytically $\underline{29}-\underline{37}$ In all of these papers clear evidence has been given that Hawking radiation is basically a low frequency phenomenon and is robust, although counterexamples were presented ${ }^{35}$ predicting strong deviation from the Hawking's result. Due to dispersion an upper threshold $\omega_{\max }$ exists in the superluminal case such that the Hawking radiation disappears for $\omega>\omega_{\max } \cdot 27,28$ For more details the reader is also referred to the review in Ref. 38 .

In the current paper we will focus on BEC or equivalently on the light in a Kerr nonlinear medium. Assuming a stationary background solution with inhomogeneous density, $\rho(\mathbf{r})$, and velocity, $\mathbf{v}(\mathbf{r})$, fields we will derive the linearized equation for the fluctuations of both fields, $\delta \rho(\mathbf{r})$ and $\delta \mathbf{v}(\mathbf{r})$, accounting for the QP. It will have a form more complicated than the Klein-Gordon equation of Ref. 4. Reducing the problem to a spatially one dimensional one, two Hermitian fields $\chi(x, t)$ and $\xi(x, t)$ are introduced, which obey two partial differential equations. We show that assuming linear dependence of the background velocity and density on the coordinate near the horizon $x=0$, the equations can be decoupled in the momentum space, and reduced to an equation for $\chi_{k}$ representing the 
density fluctuations $\delta \rho(\mathbf{r})$. Our mathematical analysis is close to that of Ref. 31, which makes use of the Laplace back transform. However, there are several formal and physical points of difference from Ref. 31, which will be discussed below. In addition the resulting dispersion relation is dictated by the GP (NLS) equation (1) (see next section) with the coordinate dependent background flow rather than chosen by the mathematical requirement of nonlinearity $\underline{26}-\underline{28}, \underline{30} \underline{-32}$ or assuming a rather general nonlinear dispersion. $\underline{25}, \underline{35}, \underline{36}$

\section{EQUATIONS FOR BEC AND LUMINOUS FLUIDS}

We start by presenting a brief overview of the basic equations controlling the dynamics of BEC or light in Kerr medium. This will allow us to make necessary definitions and introduce basic notions needed for the further analysis. Dynamics of Bose-Einstein condensate is quite successfully described by the Gross - Pitaevskii (GP) equation

$$
i \hbar \partial_{t} \Phi(\mathbf{r}, t)=-\frac{\hbar^{2}}{2 m} \nabla^{2} \Phi(\mathbf{r}, t)+U_{e x t}(\mathbf{r}) \Phi(\mathbf{r}, t)+g|\Phi(\mathbf{r}, t)|^{2} \Phi(\mathbf{r}, t)
$$

where $\Phi(\mathbf{r}, t)$ is the condensate wave function of identical Bose particles with the mass $m$, $U_{\text {ext }}(\mathbf{r})$ is an external potential, and $g$ is the parameter of the particle interaction. The equation is usually derived (see, e.g. Ref. 39) from the equation of motion for the boson field operator $\widehat{\Psi}(\mathbf{r}, t)$, which is represented in the form

$$
\widehat{\Psi}(\mathbf{r}, t)=\Phi(\mathbf{r}, t)+\widehat{\psi}(\mathbf{r}, t)
$$

so that the field operator $\widehat{\psi}(\mathbf{r}, t)$ describes fluctuations near the condensate state $\Phi(\mathbf{r})$. For small fluctuations the corresponding linearized equation reads

$$
i \hbar \partial_{t} \widehat{\psi}(\mathbf{r}, t)=\left(-\frac{\hbar^{2}}{2 m} \nabla^{2}+U_{e x t}(\mathbf{r})-\mu\right) \widehat{\psi}(\mathbf{r}, t)+2 g|\Phi(\mathbf{r})|^{2} \widehat{\psi}(\mathbf{r}, t)+g \Phi^{2}(\mathbf{r}) \widehat{\psi}^{\dagger}(\mathbf{r}, t)
$$

Usually solution $\Phi(\mathbf{r})$ of the stationary GP equation is used, which is obtained from Eq. (11) by replacing its left hand side by $\mu \Phi(\mathbf{r})$, where $\mu$ is the chemical potential.

A similar mathematical description is applied to the coherent light propagating in Kerr nonlinear medium (see, e.g., Ref. 40). The corresponding equation

$$
i \partial_{z} A=-\frac{1}{2 \beta_{0}} \widetilde{\nabla}^{2} A-\frac{\omega_{0}^{2}}{2 \beta_{0} c^{2}} \Delta n^{2}(x, y) A+\lambda|A|^{2} A .
$$


is usually called Nonlinear Schrödinger (NLS) equation. The latter is derived from the classical Maxwell equations for electromagnetic waves in a Kerr nonlinear medium. The paraxial approximation is assumed, according to which the electric component of the wave is represented in the form

$$
E=\int \frac{d \omega}{2 \pi} A(x, y ; z ; \omega) e^{-i \beta_{0} z} e^{i \omega t}
$$

where the amplitude $A$ weakly depends on the coordinate $z$ along the light propagation axis. As for its frequency dependence it is narrowly peaked around the principal frequency $\omega_{0}$. Here $\beta_{0}=k\left(\omega_{0}\right)$ where $k^{2}(\omega)=\omega^{2} \mu_{0} \varepsilon(\omega)$. The magnetic susceptibility $\mu_{0}$ is assumed to be a constant, whereas the frequency dependence of the dielectric function $\varepsilon(\omega)$ plays an important role. $c$ is the light velocity. Usually the light polarization does not change, which allows one to treat this equation as a scalar one.

Although NLS Eq.(41) is classical we can formally multiply it by $\hbar$ and get the correspondence to the quantum GP equation where the propagation distance $z$ plays the part of time, $\hbar \beta_{0}$ is the mass of a fictitious particle, which is measured in units of momentum. It is interesting to note that a quantity corresponding to energy or chemical potential should be also measured in units of momentum. It implies also that the "velocity" is dimensionless. Spacial variation $U_{e x t}(x, y)=-\hbar \frac{\omega_{0}^{2}}{2 \beta_{0} c^{2}} \Delta n^{2}(x, y)$ of the refraction index $n^{2}=\mu_{0} \varepsilon(\omega) c^{2}$ plays now the part analogous to that of the external potential in the GP equation and the field dependence of the dielectric function results in the nonlinear term in Eq.(41).

The time coordinate appears now as one of the "space" coordinates in the effective Laplacian

$$
\widetilde{\nabla}^{2}=\partial_{x}^{2}+\partial_{y}^{2} \pm \partial_{\tau}^{2}
$$

where

$$
\tau=\sqrt{\frac{1}{2 \pi|D| \beta_{0}}}\left(t-\frac{z}{v_{g}}\right),
$$

$v_{g}^{-1}=\left.\frac{\partial k(\omega)}{\partial \omega}\right|_{\omega=\omega_{0}}$ is the light group velocity in the medium and $D=\left.\frac{1}{2 \pi} \frac{\partial^{2} k(\omega)}{\partial \omega^{2}}\right|_{\omega=\omega_{0}}$ is the dispersion coefficient. The minus sign in Eq. (5) corresponds to the normal dispersion $(D>0)$ whereas the plus sign corresponds to the anomalous dispersion $(D<0)$. The hyperbolic "Laplacian" for the normal dispersion can often occur for the coherent light propagating in a Kerr medium but does not appear in the GP equation for BEC gases. 
We can always choose the frequency window within the anomalous dispersion regime such that the Laplacian keeps its standard shape for the coherent light as well. When a laser emits a pulse, $z$ is actually the position of the pulse reached during the time $z / v_{g} . \tau$ is used to describe the shape of the pulse (in the $z$ direction) in the coordinate system moving together with it.

The amplitude $A$ can be always rescaled in such a way as to make its dimension coincide with that of the wave function $\Phi$. It does not influence the linear part of Eq.(44), whereas the factor appearing in the nonlinear term can be absorbed into the parameter $\lambda$, which together with $\hbar$ then maps onto $g$ of Eq.(1), making the mapping between the two equations nearly complete.

Now we can also consider stationary solution of the NLS equation and small fluctuations around it,

$$
A(\widetilde{\mathbf{r}}, z)=\Phi(\widetilde{\mathbf{r}})+\psi(\widetilde{\mathbf{r}}, z) .
$$

where $\widetilde{\mathbf{r}}=(x, y, \tau) . \quad \psi(\widetilde{\mathbf{r}}, z)$ satisfies an equation, which maps onto Eq. (3) according to the above prescriptions with the only difference that $\psi$ in Eq.([6) is a scalar function rather than a field operator of Eq.(2).

\section{EQUATION OF MOTION FOR FLUCTUATIONS}

The hydrodynamic description of the Heisenberg equation of motion for the field operators $\widehat{\Psi}(\mathbf{r}, t)$ and $\widehat{\Psi}^{\dagger}(\mathbf{r}, t)$ follows by introducing the density operator

$$
\widehat{\rho}(\mathbf{r}, t)=m \widehat{\Psi}^{\dagger}(\mathbf{r}, t) \widehat{\Psi}(\mathbf{r}, t)
$$

and current density operator

$$
\widehat{\mathbf{j}}(\mathbf{r}, t)=\frac{1}{2 i}\left[\widehat{\Psi}^{\dagger}(\mathbf{r}, t) \nabla \widehat{\Psi}(\mathbf{r}, t)-\left(\nabla \widehat{\Psi}^{\dagger}(\mathbf{r}, t)\right) \widehat{\Psi}(\mathbf{r}, t)\right] .
$$

Both are connected by the continuity equation

$$
\partial_{t} \widehat{\rho}(\mathbf{r}, t)+\nabla \widehat{\mathbf{j}}(\mathbf{r}, t)=0
$$

Substituting Eq.(2) into Heisenberg equations of motion for $\widehat{\Psi}(\mathbf{r}, t)$ and $\widehat{\Psi}^{\dagger}(\mathbf{r}, t)$, using $\Phi(\mathbf{r}, t)=f(\mathbf{r}, t) \exp [-i \varphi(\mathbf{r}, t)]$ and neglecting fluctuations one obtains hydrodynamic equations

$$
\frac{\partial}{\partial t} \rho+\nabla \cdot[\rho \mathbf{v}]=0
$$




$$
\frac{\partial}{\partial t} \mathbf{v}+\frac{1}{2} \nabla \mathbf{v}^{2}=-\frac{1}{m} \nabla\left[U_{q u}+U_{e x t}+g f^{2}\right]
$$

for the density $\rho(\mathbf{r}, t)=m f^{2}(\mathbf{r}, t)$ and velocity $\mathbf{v}(\mathbf{r}, t)=-\frac{\hbar}{m} \nabla \varphi(\mathbf{r}, t)$; both $f$ and $\varphi$ are real functions. These two equations are equivalent to the GP equation (1). Here

$$
U_{q u}=-\frac{\hbar^{2}}{2 m} \frac{\nabla^{2} f}{f}
$$

is QP. This hydrodynamic representation of the basic equations allows us to think about the BEC fluid or luminous fluid, respectively.

We now consider small fluctuations of these two fields with respect to a stationary solution $\Phi_{0}(\mathbf{r})=f_{0}(\mathbf{r}) \exp \left[-i \varphi_{0}(\mathbf{r})\right]$. It follows from Eq.(17) that

$$
\nabla \cdot\left[\rho_{0}(\mathbf{r}) \mathbf{v}_{0}(\mathbf{r})\right]=0
$$

where $\rho_{0}(\mathbf{r})=m f_{0}^{2}(\mathbf{r})$ and $\mathbf{v}_{0}(\mathbf{r})=-\frac{\hbar}{m} \nabla \varphi_{0}(\mathbf{r})$. To describe the fluctuations we introduce the dimensionless quantities

$$
\begin{aligned}
& \chi=\frac{1}{f_{0}}\left[e^{-i \varphi_{0}} \psi^{\dagger}+e^{i \varphi_{0}} \psi\right], \\
& \xi=\frac{1}{2 i f_{0}}\left[e^{-i \varphi_{0}} \psi^{\dagger}-e^{i \varphi_{0}} \psi\right],
\end{aligned}
$$

which are field operators in the case of BEC or classical scalar fields in the case of coherent light. Therefore we skipped the hats. These quantities are connected with the density and velocity fluctuations by the relations

$$
\begin{aligned}
\delta \mathbf{v}(\mathbf{r}, t) & =-\frac{\hbar}{m} \nabla \xi(\mathbf{r}, t) \\
\delta \rho(\mathbf{r}, t) & =\rho_{0}(\mathbf{r}) \chi(\mathbf{r}, t)
\end{aligned}
$$

Then it is straightforward to show that Eq.(2) and its Hermitian conjugate lead to

$$
\begin{gathered}
\widehat{D} \chi-\frac{\hbar}{m} \frac{1}{f_{0}^{2}} \nabla\left(f_{0}^{2} \nabla \xi\right)=0, \\
\widehat{D} \xi+\frac{\hbar}{4 m} \frac{1}{f_{0}^{2}} \nabla\left(f_{0}^{2} \nabla \chi\right)-\frac{1}{\hbar} g f_{0}^{2} \chi=0 .
\end{gathered}
$$

where $\widehat{D}=\partial_{t}+\mathbf{v}_{0} \cdot \nabla$. These equations also follow from Eqs.(7) and (8) in the linear order in $\delta \rho$ and $\delta \mathbf{v}$. While deriving equations (13), we used Eq.(10).

It is instructive to indicate that Eqs. (13) are Euler - Lagrange equations (see also discussion in Appendix of Ref. 28) corresponding to the Lagrangian density 


$$
\mathcal{L}=\frac{1}{2} f_{0}^{2}(\chi \dot{\xi}-\xi \dot{\chi})+\frac{1}{2} f_{0}^{2} \mathbf{v}_{0} \cdot(\chi \nabla \xi-\xi \nabla \chi)-\frac{1}{2 \hbar} g f_{0}^{4} \chi^{2}-\frac{\hbar}{2 m} f_{0}^{2}(\nabla \xi)^{2}-\frac{\hbar}{8 m} f_{0}^{2}(\nabla \chi)^{2}
$$

This Lagrangian density allows us also to define canonical momenta

$$
p_{\chi}=\frac{\delta}{\delta \dot{\chi}}\left[\mathcal{L}-\frac{1}{2} \partial_{t}\left(f_{0} \chi \xi\right)\right]=-f_{0}^{2} \xi, \quad p_{\xi}=\frac{\delta}{\delta \dot{\xi}}\left[\mathcal{L}+\frac{1}{2} \partial_{t}\left(f_{0} \chi \xi\right)\right]=f_{0}^{2} \chi
$$

for each of the fields $\chi$ and $\xi$. Together they form two canonical pairs, each of which can be used to derive Hamiltonian. The Hamiltonian density for these two cases can be derived as

$$
\begin{gathered}
\mathcal{H}=-f_{0} \dot{\chi} \xi-\left[\mathcal{L}-\frac{1}{2} \partial_{t}\left(f_{0} \chi \xi\right)\right]=f_{0} \chi \dot{\xi}-\left[\mathcal{L}+\frac{1}{2} \partial_{t}\left(f_{0} \chi \xi\right)\right]= \\
-\frac{1}{2} f_{0}^{2} \mathbf{v}_{0} \cdot(\chi \nabla \xi-\xi \nabla \chi)+\frac{1}{2 \hbar} g f_{0}^{4} \chi^{2}+\frac{\hbar}{2 m} f_{0}^{2}(\nabla \xi)^{2}+\frac{\hbar}{8 m} f_{0}^{2}(\nabla \chi)^{2} .
\end{gathered}
$$

Although the derivation (16) depends on the choice of the canonical pair, the final Hamiltonian does not depend on it. Eqs. (13) are now just the pair of Hamilton equations of motion for the Hamiltonian density (16). The above manipulations with adding or subtracting a full derivative indicate that the variations of the Lagrangian with respect to $\dot{\chi}$ and $\dot{\xi}$ are not uniquely defined. This feature of the Lagrangian (14) does not however effect the Euler- Lagrange equations (13) but is important when defining the canonical momentum and Hamiltonian. It means that we have to choose which of the functions $\chi$ or $\xi$ plays the part of the canonical coordinate, then the other one will enter the definition of the conjugate canonical momentum. It is emphasized that we can use only one of these pairs at a time, but not both of them simultaneously. The physical result is independent of the choice.

If $\xi$ is chosen as the canonical coordinate then the quantization condition reads

$$
\left[p_{\xi}(\mathbf{r}, t), \xi\left(\mathbf{r}^{\prime}, t\right)\right]=\left[f_{0}^{2} \chi(\mathbf{r}, t), \xi\left(\mathbf{r}^{\prime}, t\right)\right]=\hbar \delta\left(\mathbf{r}-\mathbf{r}^{\prime}\right)
$$

where $[\cdots, \cdots]$ denotes the commutator of two operators. The other choice of the canonical pair would obviously yield the same quantization condition. One can readily see that the quantization condition (17) in the BEC case is consistent with the definition (11), where $\psi^{\dagger}$ and $\psi$ are Bose field operators. In case of the coherent light when these are scalar functions, Eq. (17) allows us to quantize them thus removing the last remaining difference between BEC and luminous liquid. 
We may extend the definition of the fields $\chi$ and $\xi$ and consider them complex. Then the Lagrangian density (14) can be represented in the form

$$
\mathcal{L}=\frac{i}{4} f_{0}^{2}\left[\vartheta^{\dagger} \sigma_{y}(\widehat{D} \vartheta)-\left(\widehat{D} \vartheta^{\dagger}\right) \sigma_{y} \vartheta\right]-\frac{1}{2 \hbar} g f_{0}^{4} \vartheta^{\dagger}\left(1+\sigma_{z}\right) \vartheta-\frac{\hbar}{4 m} f_{0}^{2}\left(\nabla \vartheta^{\dagger}\right) \nabla \vartheta
$$

where the two component field

$$
\vartheta=\left(\begin{array}{c}
\frac{1}{\sqrt{2}} \chi \\
\sqrt{2} \xi
\end{array}\right)
$$

has been introduced.

The corresponding equation of motion reads

$$
\frac{i}{2} f_{0}^{2} \sigma_{y} \dot{\vartheta}+\frac{i}{2} f_{0}^{2} \mathbf{v}_{0} \cdot \sigma_{y} \nabla \vartheta-\frac{1}{2 \hbar} g f_{0}^{4}\left(1+\sigma_{z}\right) \vartheta+\frac{\hbar}{4 m} \nabla\left(f_{0}^{2} \nabla \vartheta\right)=0
$$

It is obviously equivalent to two Eqs.(13). Now we multiply Eq.(20) by $2 \vartheta^{\dagger}$ from the left, write also a Hermitian conjugate equation and take their difference. The result is

$$
\partial_{t}\left(f_{0}^{2} \vartheta^{\dagger} \sigma_{y} \vartheta\right)+\nabla\left(f_{0}^{2} \mathbf{v}_{0} \vartheta^{\dagger} \sigma_{y} \vartheta\right)-\frac{i \hbar}{2 m} \nabla\left[f_{0}^{2}\left[\vartheta^{\dagger} \nabla \vartheta-\left(\nabla \vartheta^{\dagger}\right) \vartheta\right]\right]=0
$$

We may conclude that the quantity $\varrho=f_{0}^{2} \vartheta^{\dagger} \sigma_{y} \vartheta$ plays the role of the density of this two component field, whereas

$$
\mathbf{j}=f_{0}^{2} \mathbf{v}_{0} \vartheta^{\dagger} \sigma_{y} \vartheta-i \frac{\hbar}{2 m} f_{0}^{2}\left[\vartheta^{\dagger} \nabla \vartheta-\left(\nabla \vartheta^{\dagger}\right) \vartheta\right]
$$

is the corresponding current. The integral

$$
\int d^{3} r f_{0}^{2} \vartheta^{\dagger} \sigma_{y} \vartheta=-i \int d^{3} r f_{0}^{2}\left(\chi^{*} \xi-\xi^{*} \chi\right)
$$

is a conserved quantity, which can be used in order to normalize solutions (eigenfunctions) of Eq.(13) or to define a scalar product of two such solutions. Similarly to the well-known Klein-Gordon norm, Eq.(22) is not positively defined. Positive and negative frequencies are considered separately in order to resolve this problem in the case of the Klein - Gordon equation (see, e.g. Ref. 3). A similar procedure will be applied in this case as well. This property will be important for the further discussion. 


\section{EIGENFUNCTIONS}

\section{A. Bogoliubov spectrum}

As an example we first may consider a homogeneous background solution $\Phi(\mathbf{r})$, in which only the phase may linearly depend on the coordinate, i.e. both the density $\rho_{0}=m f_{0}^{2}$ and the velocity $m \mathbf{v}_{0}=-\hbar \nabla \varphi_{0}$ are constants. Then by solving the second equation in Eq.(13) for $\chi$ and substituting the result in the first one this system reduces to a linear partial differential equation with constant coefficients:

$$
\widehat{D}^{2} \xi-\frac{g}{m^{2}} \rho_{0} \nabla^{2} \xi+\frac{1}{\hbar^{2}}\left(\frac{\hbar^{2}}{2 m} \nabla^{2}\right)^{2} \xi=0 .
$$

It straightforwardly leads to the celebrated Bogoliubov spectrum

$$
\left[\omega-\mathbf{v}_{0} \cdot \mathbf{k}\right]^{2}=\bar{s}^{2} k^{2}\left[1+\frac{k^{2} l_{h}^{2}}{2}\right] \equiv \Omega^{2}(k)
$$

in the coordinate system moving with the constant velocity $\mathbf{v}_{0}$. The quantity $\bar{s}^{2}=\frac{g \rho_{0}}{m^{2}}$ is obviously the sound velocity in the long wave limit and $l_{h}^{2}=\hbar^{2} /\left(2 m g f_{0}^{2}\right)=\hbar^{2} /\left(2 m^{2} \bar{s}^{2}\right)$ is

the healing length in BEC and the nonlinearity length $l_{h}^{2}=1 /\left(2 \beta_{0} \lambda|A|^{2}\right)$ in optics. These lengths are determined by comparing the QP $U_{q u}$ with the nonlinear term in the equation of motion. Eq. (24) demonstrates that the propagation at high frequencies corresponds to the so called superluminal case. For $l_{h}=0$, i.e. neglecting $\mathrm{QP}, \Omega(k)$ reduces to a linear dispersion law. It is interesting to indicate that the Bogoliubov spectrum of excitations appears not only in the quantum BEC system, for which it has been originally proposed, but also in the case of classical electromagnetic wave propagating in the Kerr medium.

Eq.(24) has four solutions, which for $\mathbf{v}_{0}=0$ read

$$
k^{2}=-\frac{1}{l_{h}^{2}}\left(1 \pm \sqrt{1+\frac{2 \omega^{2} l_{h}^{2}}{\bar{s}^{2}}}\right)
$$

Two of the four solutions (- sign in Eq.(25) $)$ are real and correspond to two plane waves moving in the mutually opposite directions. The other two solutions (+ sign in Eq.(25]) are imaginary and correspond to excitations growing or decaying exponentially on the scale $l_{h}$. They are usually of a minor physical importance. The situation may change for the supersonic regime when four real solutions are possible, provided $\omega<\omega_{\max } .^{27,28}$ 


\section{B. Hydrodynamic approximation}

The hydrodynamic approximation, applied in many cases, assumes that the QP $U_{q u}$ in Eq.(8) is neglected. It is justified when the QP is small as compared to the interaction energy, $U_{q u} \ll g f_{0}^{2}$, i.e. on the large scales $|x| \gg l_{h}$. Now again it is possible to eliminate the variable $\chi$ in Eq. (13), which is then reduced to the equation

$$
\frac{\hbar^{2}}{g f_{0}^{2}}\left\{\left[\partial_{t}^{2}+\partial_{t} \mathbf{v}_{0} \cdot \nabla\right] \xi+\nabla\left(\cdot \mathbf{v}_{0} \partial_{t} \xi\right)+\nabla\left(\cdot \mathbf{v}_{0}\left(\mathbf{v}_{0} \cdot \nabla\right)\right) \xi-\nabla\left(s^{2} \nabla\right) \xi\right\}=0 .
$$

for the phase fluctuation field $\xi$.

This equation has the form of a Klein-Gordon equation in a curved space, which has been discussed many times starting from the seminal paper by Unruh $\underline{4}^{4}$ (see also Refs. 8, 12,33,34). In particular, we may apply it to the situation, which takes place in the Laval nozzle (Fig. 11), creating a transonic flow of a luminous fluid. $\underline{\underline{12}}$ In this case we may reduce the problem to $1+1$ dimensions keeping the time coordinate and one spatial coordinate $x$ along the streamline crossing the Mach horizon at $x=0$. In its narrow vicinity the velocity is a linear function of the coordinate, $v_{0}(x) \approx \bar{s}(1+\alpha x)$. Since the density $\rho_{0}(x) v_{0}(x)=\frac{m^{2}}{g} s^{2}(x) v_{0}(x)$ of the flow reaches its maximum at the throat of the nozzle we have $s(x) \approx \bar{s}\left(1-\frac{1}{2} \alpha x\right)$. Therefore the functions $v(x)$ and $s(x)$ cannot be chosen independently unless we allow for the $x$ dependence of the nonlinearity coefficient. $\underline{\underline{28}}$ Then Eq.(26) becomes

$$
\left\{\partial_{\tau}^{2}+2(1+\alpha x) \partial_{\tau} \partial_{x}+\alpha \partial_{\tau}+3 \alpha \partial_{x}\left(x \partial_{x}\right)\right\} \xi=0
$$

to within the linear terms in $x$, where the variable $\tau=t \bar{s}$ is used. This equation has two solutions, which at $|x| \rightarrow 0$ behave as

$$
\xi_{1} \propto e^{\gamma_{0} \ln x-i \omega t}, \quad \xi_{2} \propto e^{i k x-i \omega t}
$$

where $\gamma_{0}=i \frac{2 \omega}{3 \bar{s} \alpha}$ and $k(\omega)=\frac{\omega}{\bar{s}} \frac{\omega+i \bar{s} \alpha}{2 \omega-3 i \bar{s} \alpha}$. Correspondingly we have also two eigenfunctions for the density fluctuations

$$
\chi_{1} \propto e^{\left(\gamma_{0}-1\right) \ln x-i \omega t}, \quad \chi_{2} \propto e^{i k x-i \omega t}
$$

These two solutions $\frac{12,33}{}$ are the former propagating plane waves in the Bogoliubov spectrum strongly distorted by the accelerating background flow. The solution $\xi_{1}$ corresponds to the excitation "attempting" to propagate against the flow in the vicinity of the 
Mach horizon, which cuts it into two parts moving in the opposite directions from the horizon. The solution $\xi_{2}$ is a fluctuation propagating with the flow and therefore have a shape quite similar to that of a plane wave. In the high frequency limit these modes propagate with the double sound velocity.

Two remarks are in order: The exponent $\gamma_{0}$ here differs from the corresponding exponent in Ref. 12 by a factor of two due to the misprint in that paper. This exponent, as well as the Hawking temperature to be obtained below, differs also from the exponent in Ref. 33,34 by a numerical factor due to the fact that here we take into account the coordinate dependence both of the velocity of the flow and of the local sound velocity.

An important feature of these two solutions is that they behave completely differently at $|x| \rightarrow 0$. The solution $\xi_{2}$ is a smooth function in this region meaning that the corrections due to the neglected QP, proportional to the second derivative of $\xi_{2}$ are small and the above approximation is quite sufficient. Completely different situation takes place when dealing with the solution $\xi_{1}$, which is singular in the limit $|x| \rightarrow 0$. The corrections to $\xi_{1}$ due to the QP diverge at $|x| \rightarrow 0$ and a more refined procedure is necessary in order to regularize the behavior of $\xi_{1}$ in this limit.

\section{Regularization}

As indicated above the eigenfunctions $\xi_{1}$ and $\chi_{1}$, obtained in the hydrodynamic approximation, (28) and (29), have a singularity (branching point) on the horizon at $x=0$. This type of behavior is typical of the eigenfunctions of the Klein-Gordon equation in the vicinity of the horizon of a real black hole (see, e.g., Ref. 43 and references therein) and leads to the celebrated Hawking radiation. Regularization in this case requires knowing the physics at the Planck length scale (see, e.g. Ref. 33). In the systems discussed here the part of Planck length is played by $l_{h}$, which is the healing length for BEC or nonlinearity length for the luminous liquid. In order to regularize $\chi_{1}, \xi_{1}$ near the horizon we have to take into account the QP in Eq. (13).

We apply now the same assumptions concerning the coordinate dependence of the velocity and density near the Laval nozzle throat as in the previous subsection. Using the Laplace representation

$$
\chi(x, \tau)=e^{-i \nu \tau} \int_{C} d k \chi_{k} e^{i k x}
$$


where $\nu=\omega / \bar{s}$, and the contour $C$ is chosen such that the integral converges,,$\underline{41}$ then Eqs. (13) become

$$
\left\{\begin{array}{c}
(-i \omega+i \bar{s} k) \chi_{k}-\alpha \bar{s} \partial_{k}\left(k \chi_{k}\right)+i \alpha k \frac{\hbar}{m} \xi_{k}+\frac{\hbar}{m} k^{2} \xi_{k}=0 \\
-\frac{\hbar^{2}}{4 m}\left[i \alpha k+k^{2}\right] \chi_{k}-m \bar{s}^{2}\left(\chi_{k}-i \alpha \partial_{k} \chi_{k}\right)+\hbar\left[(-i \omega+i \bar{s} k) \xi_{k}-\alpha \bar{s} \partial_{k}\left(k \xi_{k}\right)\right]=0 .
\end{array}\right.
$$

where the terms $O\left(\alpha^{2} / k^{2}\right)$ have been omitted. This approximation is equivalent to considering a region $\alpha|x| \ll 1$ near the horizon. The representation (30) is especially convenient. It allows us to solve explicitly the first equation,

$$
\xi_{k}=\frac{m}{\hbar} \frac{(i \omega-i \bar{s} k) \chi_{k}+\alpha \bar{s} \partial_{k}\left(k \chi_{k}\right)}{i \alpha k+k^{2}},
$$

and substitute the result into the second equation. We then get equation

$$
\partial_{k} \ln \chi_{k}=\frac{1}{i \alpha k(2 \nu-3 k-i \alpha)}\left[-\frac{l_{h}^{2}}{2}\left(i \alpha k+k^{2}\right)^{2}-k^{2}+(\nu-k)^{2}\right]
$$

for the function $\chi_{k}$. Two comments are in order with respect to the approach taken in Ref. 31 where the Laplace representation has also been used. Although Eq. (32) has the structure similar to that of Eq. (23) in Ref. 31, it is certainly different. Most important is that the r.h.s. of Eq. 32 has two poles instead of a single pole at $k=0$ (Ref. 31 uses $k=-i s)$. This difference stems from neglecting one of the terms in equation (12) in Ref. 31. However, as we will see below, it is crucial for our analysis not to neglect this term. An attention is also drawn to the fact that the r.h.s. of Eq. 32 involves a cubic term with purely imaginary coefficient.

Eq. (32) can be readily solved to within a term independent of $k$,

$$
\ln \chi_{k}=\gamma_{1} \ln (k)+\gamma_{2} \ln \left(k-\frac{2}{3} \nu-\frac{i}{3} \alpha\right)+\Lambda(k, \nu)
$$

where

$$
\gamma_{1}=\frac{1}{4}-\frac{i \nu}{2 \alpha}, \quad \gamma_{2}=-\frac{1}{4}-i \frac{1}{6 \alpha} \nu-\frac{4 i}{81 \alpha} l_{h}^{2} \nu^{3}+\frac{14}{81} l_{h}^{2} \nu^{2}
$$

and the $l_{h}$ dependent part is given by

$$
\Lambda(k, \nu)=\frac{l_{h}^{2}}{\alpha}\left\{-\frac{i}{18} k^{3}+\frac{5}{36} \alpha k^{2}-\frac{i}{18} \nu k^{2}-\frac{2 i}{27} \nu^{2} k+\frac{4}{27} \nu \alpha k\right\} .
$$

The real space solution of Eq. (13) is finally obtained by the back Laplace transform

$$
\chi(x, t)=e^{-i \nu \tau} \int_{C} d k k^{\gamma_{1}}\left(k-\frac{2}{3} \nu-\frac{i}{3} \alpha\right)^{\gamma_{2}} \exp \{\Lambda(k, \nu)+i k x\} .
$$


This integral is calculated in the way similar to that used for the calculation of Airy functions in Ref. 41. First, we have to find the sectors of convergency of this integral in the Riemann plane for the complex variable $k$, which are controlled by the term proportional to $k^{3}$ in Eq. (34). Hence the condition $\operatorname{Im} k^{3}<0$ must be required. It holds in the three sectors: $2 \pi / 3>\arg k>\pi / 3,4 \pi / 3>\arg k>\pi$ and $2 \pi>\arg k>5 \pi / 3$, shown in Figure 2. The integrand's function has also two branching points at $k=0$ and $k=2 \nu / 3+i \alpha / 3$, which result in two cuts shown in Figure 2. Due to these two cuts there are five possible integration contours $C_{1}$ to $C_{5}$ and any other contour may be represented as a combination of these five. They provide us with five different results for the integral (35), however the sum of these five integrals is zero due to the Cauchy theorem. As already mentioned above a similar approach to the problem was applied in Ref. 31 with nearly the same configuration of contours as in Fig. 2. However, the corresponding function in Ref. 31 has only one branching point. But to have four independent solutions we need two branching points rather than one. They are also important for Eq. (37) to be derived below.

The corresponding four integrals may be calculated by the steepest decent method. Then each of these integrals will be a linear combination of the contributions due to the four saddle points, determined by the equation

$$
\partial_{k} \ln \chi_{k}=-i x
$$

Alternatively we may also choose the integrals due to the individual saddle points as linearly independent solutions. Substituting $\partial_{k} \ln \chi_{k}$ from Eq. (32) into Eq. (36) we get after some manipulations the $x$ dependent dispersion relation

$$
\frac{l_{h}^{2}}{2}\left(i \alpha k+k^{2}\right)^{2} \bar{s}^{2}+k^{2} s^{2}(x)=[\omega-k v(x)]^{2}=\Omega^{2}(k, \omega)
$$

where we remind that $v(x) \approx \bar{s}(1+\alpha x), s(x) \approx \bar{s}\left(1-\frac{1}{2} \alpha x\right)$ and $\omega=\bar{s} \nu$. Here some terms of the order $O\left((\alpha / k)^{2}\right)$ and $O\left((\alpha x)^{2}\right)$ are kept only for the sake of a compact presentation. However only the terms up to the first order are meaningful.

Equation (37) is rather similar to Eq. (6) in Ref. 31 and Eq. (23) in Ref. 28. Nevertheless we have to indicate important differences. Those equations relate to the regions $\alpha|x| \gg 1$ far from the horizon where it is assumed that the flow velocity reaches its supersonic or subsonic limits, and the corresponding velocities, $v_{\mp}$, do not depend on the coordinate $x$. Our equation (37) is deduced for the region close to the horizon 


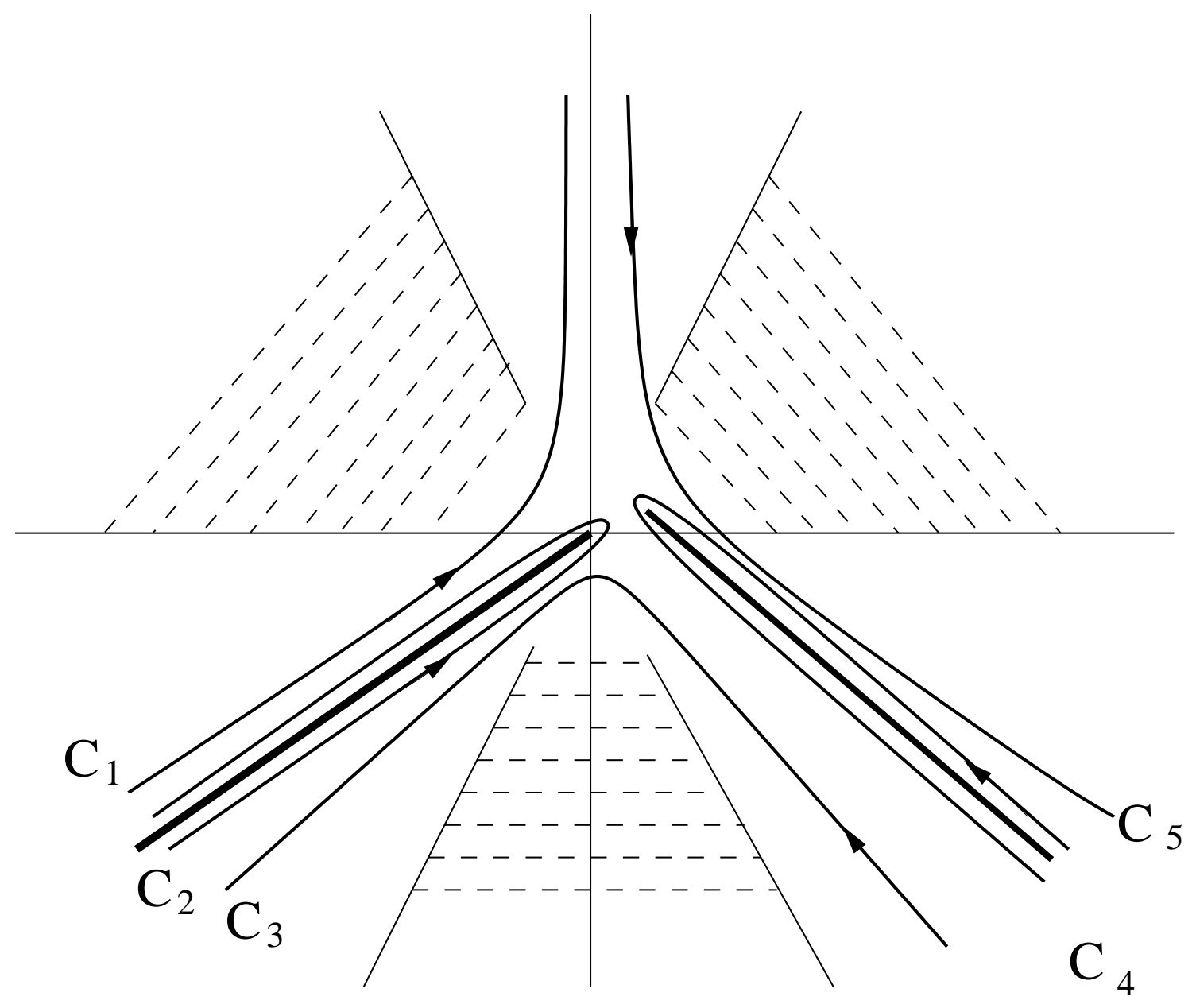

FIG. 2: Five possible contours in the complex $k$ plane for calculation of the integral (35). The integral diverges in the dashed sectors. The contours lie in the sectors of convergency and also go around the two cuts, which start from the two branching points at $k=0$ and $k=\frac{2}{3} \nu+\frac{i}{3} \alpha$.

$\alpha|x|<0$ and both the flow and sound velocities depend on the coordinate. It also contains an imaginary correction in the quartic term.

An interesting insight may be obtained from this equation by a graphical analysis, which is similar in some aspects to the one carried out in Refs. 30,31 and particularly in Ref. 28. Let us neglect the imaginary correction to the quartic term, which can be taken into account as a perturbation, and plot both branches of the function $\Omega(k, \omega)$ crossed by the three straight lines $\omega-k v(x)$ for three different values of $|x|$, Fig. 3, We also do not take into account the $x$ dependence of the sound velocity, since it would have overloaded the graphical presentation without changing any conclusions. All the three 


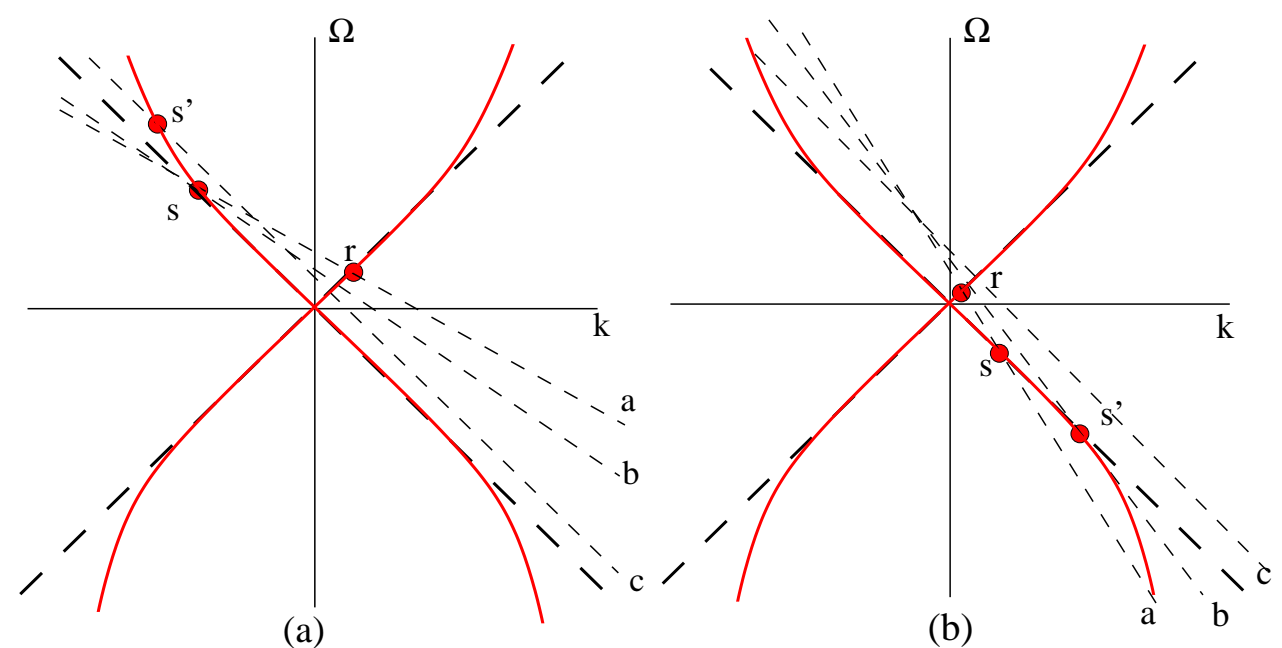

(a)

(b)

FIG. 3: (Color online) Graphical analysis of equation (37). Two branches of the function $\Omega(k, \omega)$ are plotted in red in figures (a) and (b). They are crossed by straight dashed lines $\omega-k v(x)$ for three values of $x<0$ ((a) - subsonic regime) and three values of $x>0$ ((b) - supersonic regime). The full circles show the most important solutions of the equation. The long dashed lines show the function $\Omega(k, \omega)$ in the absence of the dispersion, i.e. $l_{h}=0$.

lines are plotted for a fixed frequency $\nu$ but decreasing distance $|x|$ from the horizon on both the subsonic side (Fig. 3a) and the supersonic side (Fig. 3b). The line (c) in both cases corresponds to $|x|=0$ when $v(x) \rightarrow \bar{s}$.

In the subsonic region (Fig. 3a) where $x<0$ we always have two real solutions $r$ and $s$, with and without dispersion. It is interesting to indicate that in the absence of the dispersion, i.e in the absence of the quartic term in Eq. (37) when $l_{h} \rightarrow 0$ and when the two branches of $\Omega(k, \omega)$ are just two straight lines with the slope one in dimensionless units (long dashed lines in Fig. 3), the $s$ solution moves to infinity $\left(k_{s} \rightarrow-\infty\right)$ as $x \rightarrow 0$. The dispersion changes this behavior so that $k_{s}$ tends to a finite value in this limit (going from line (a) to (b) and then to (c)) at the point (s') where the (c) straight line crosses the $\Omega(k, \omega)$ curve.

In the supersonic case, the straight line (a) crosses the curve $\Omega(k, \omega)$ in four points producing three right movers and one left mover (not all are visible in Fig. 31). It is important to emphasize that two of these solutions, i.e. both right movers $(r)$ and $(s)$ exist even in the absence of the dispersion. If we move closer to the horizon, $|x| \rightarrow 0$, the slope of the straight lines tends to that corresponding to the sound velocity $($ line $(\mathrm{c}))$. In 
the absence of the quartic term in Eq. (37) the $s$ solution moves to infinity as we approach the horizon $x \rightarrow 0$.

Two solutions (both for subsonic and supersonic case)

$$
k_{s}=\frac{2 \nu}{3 \alpha x}, \quad k_{r}=\frac{2 \nu}{4-\alpha} .
$$

are obtained directly from Eq. (37) at $l_{h}=0$. The saddle point $k_{r}$ leads us straightforwardly to the right mover eigenfunction $\chi_{2}$ of Eq.(29), which will be now denoted by $\chi_{r}$. As for the $k_{s}$ point, the integral (35) converges in a very broad range of $k$ values. We may introduce the new variable $z=k x$ and write the integral (35) in the approximate form

$$
\chi_{s}(x, \tau) \approx x^{-\left(\gamma_{1}+\gamma_{2}+1\right)} e^{-i \nu \tau} \int d z z^{\gamma_{1}+\gamma_{2}} \exp \{i z\}
$$

where neglecting $\Lambda(k, \nu)$ in the exponent of the integrand is justified as long as $x \gg l_{h}$. (A stricter inequality will be given below). The validity of this equation is limited also by the inequality $x \ll \min \{1 / \nu, 1 / \alpha\}$. As a result we get the singular eigenfunction

$$
\chi_{s} \propto x^{\gamma-1}
$$

in which the parameter

$$
\gamma=-\gamma_{1}-\gamma_{2}=\frac{2 i \nu}{3 \alpha}+\frac{4 i}{81} \frac{l_{h}^{2} \nu^{3}}{\alpha}-\frac{2}{27} l_{h}^{2} \nu^{2}
$$

differs from the parameter $\gamma_{0}$ in Eqs. (28) and (29) for $\xi_{1}$ and $\chi_{1}$, respectively, since it contains small corrections due to the QP. That is why the notation $\chi_{s}$ is used instead of $\chi_{1}$. This type of singular behavior of the eigenfunction typically occurs near the horizon and is a crucial ingredient in formation of the Hawking radiation ( see, e.g., Refs. 42,43).

Accounting for the quartic term in Eq. (37) introduces important changes. While moving towards the horizon, $|x| \rightarrow 0$, for a given frequency we rotate the straight lines in Fig. 3 so that the line $(a)$ becomes $(b)$ when two real solutions merge (shown as the point s' in Fig. $3 \mathrm{~b}$ ), and then disappear (become complex) (c). It actually means that the singular behavior (38) of the eigenfunction $\chi_{s}$ holds for not two small values of $|x|$ and becomes regularized closer to the horizon. Here a remark is in order. It was indicated in Refs. 27,28 that there is a maximal frequency $\omega_{\max }$, above which this pair of solutions does not appear at all, which makes the Hawking radiation impossible. This condition was obtained by considering the behavior of the eigenfunctions in the asymptotic region 
$x \rightarrow \infty$ far from the horizon where the flow velocity becomes constant. Our equation (37) allows for a smooth interpolation between these two regions. It is clear that if we start at $\omega>\omega_{\max }$ in the supersonic asymptotic region those two solutions do not show up, and then moving closer to the horizon, leading to a variation of the sound and flow velocities, the singular solution $s$ will have no chance to appear in the close vicinity of the horizon as well. If in contrast $\omega<\omega_{\max }$, both solutions exist for $x \rightarrow \infty$. Now approaching the horizon a critical distance $x_{*}(\omega)$ exists such that that these solutions disappear for $x<x_{*}(\omega)$.

We may put the above pattern on a more quantitative basis. In order to find the behavior of $\chi_{s}$ in the nearest vicinity of the horizon we substitute $k_{s} \sim x^{-1}$ in the dimensionless exponent $\Lambda$ in Eq.(35) and obtain that the cubic term in Eq. (34) (former quartic term in Eq.(32)) becomes large when

$$
|x|<l_{r}=\frac{l_{h}}{\left(l_{h} \alpha\right)^{1 / 3}} .
$$

In this region the convergency of the integral (35) is controlled by the cubic term in $\Lambda$ and the result remains finite and not singular at $|x| \rightarrow 0$, i.e. the function $\chi_{s}$ is regularized at $|x|<l_{r}$. In order to demonstrate this it is sufficient to calculate the integral

$$
\begin{gathered}
i \int_{0}^{\infty} z^{-\gamma} \exp \left(-\frac{1}{18} l_{r}^{3} z^{3}-z x\right) d z= \\
=\frac{3}{l_{r}} \exp \left(\frac{i}{2}-\gamma \pi\right)\left(-i \frac{l_{r}^{3}}{18}\right)^{-\frac{1}{3}-\gamma}\left\{-2\left(-i \frac{1}{18}\right)^{\frac{2}{3}} \Gamma\left(\frac{1-\gamma}{3}\right){ }_{1} F_{2}\left(\frac{1-\gamma}{3} ; \frac{1}{3}, \frac{2}{3} ;-\frac{2 x^{3}}{3 l_{r}^{3}}\right)+\right. \\
2 i\left(-i \frac{1}{18}\right)^{\frac{1}{3}} \frac{x}{l_{r}} \Gamma\left(\frac{2-\gamma}{3}\right){ }_{1} F_{2}\left(\frac{2-\gamma}{3} ; \frac{2}{3}, \frac{4}{3} ;-\frac{2 x^{3}}{3 l_{r}^{3}}\right)+ \\
\left.\frac{x^{2}}{l_{r}^{2}} \Gamma\left(\frac{3-\gamma}{3}\right){ }_{1} F_{2}\left(\frac{3-\gamma}{3} ; \frac{4}{3}, \frac{5}{3} ;-\frac{2 x^{3}}{3 l_{r}^{3}}\right)\right\}
\end{gathered}
$$

whose integrand is the approximate integrand of (35) at large $k$. It represents the integral (35) over the upper half of the contour $C_{1}$ or $C_{5}$. Here ${ }_{1} F_{2}(a ; b, c ; x)$ is a hypergeometric function and $\Gamma(x)$ is the Euler Gamma-function. The other integrals corresponding to other contours or their parts can be obtained by changing the relevant phases. This integral tends to the finite limit

$$
\frac{1}{3}\left(-\frac{i l_{r}^{3}}{18}\right)^{-\frac{1-\gamma}{3}} \exp \left(-\frac{i}{2} \gamma \pi\right) \Gamma\left(\frac{1-\gamma}{3}\right)
$$


for $x \rightarrow 0$, in fact for $|x| \ll l_{r}$. The derivatives with respect to $x$ in this limit can be also calculated, resulting in a regular Taylor expansion. One can also readily see that this function may have zeros at complex $x$.

It is emphasized that the integral (41) depends on the regularization length $l_{r}$ rather than on the healing length $l_{h}$. It is also interesting that typically $l_{h} \alpha<1$ so that regularization starts at the scale larger than the healing length, $l_{r}>l_{h}$.

\section{HAWKING RADIATION FROM THE MACH HORIZON.}

In order to calculate the frequency spectrum of the Hawking radiation emanating from the Mach horizon we will apply the approach similar to that proposed by Damour etal. $\underline{42,43}$ The central point in this approach is the calculation of the norm of a straddled fluctuation, which clearly demonstrates how a negative frequency state is cut into negative and positive frequency states propagating in the opposite directions from the horizon. In our case we have to calculate the norm (22) for a system with variation in the $x$ direction only:

$$
<\vartheta_{s}, \vartheta_{s}>=\int d x \varrho_{s}(x)=-i \int d x f_{0}^{2}\left(\chi_{s}^{*} \xi_{s}-\xi_{s}^{*} \chi_{s}\right)
$$

for the pair of eigenfunctions $\left(\xi_{s}, \chi_{s}\right)$. We have to deal here with the two component function (19), of which $\chi_{s}$ calculated above is only one component. The second component $\xi_{s}$ can be found now, say, by solving Eq. (13). Neglecting again the contribution of the $\mathrm{QP}$ in Eq. (13) we get

$$
\chi_{s}=\frac{\hbar}{g f_{0}^{2}} \widehat{D} \xi_{s}
$$

so that

$$
\xi_{s} \propto x^{\gamma}
$$

for $\min \{1 / \nu, 1 / \alpha\} \gg|x| \gg l_{r}$.

The two component field density and current density are

$$
\begin{gathered}
\varrho_{s}=-i\left\{\left[\left(\partial_{t} \xi_{s}^{*}\right) \xi_{s}-\xi_{s}^{*} \partial_{t} \xi_{s}\right]+v_{0}(x)\left[\left(\partial_{x} \xi_{s}^{*}\right) \xi_{s}-\xi_{s}^{*} \partial_{x} \xi_{s}\right]\right\} \\
j_{s}=i\left\{v_{0}\left(\xi_{s}^{*} \partial_{t} \xi_{s}-\left(\partial_{t} \xi_{s}^{*}\right) \xi_{s}\right)+\left(v_{0}^{2}(x)-s^{2}(x)\right)\left(\xi_{s}^{*} \partial_{x} \xi_{s}-\left(\partial_{x} \xi_{s}^{*}\right) \xi_{s}\right)\right\} .
\end{gathered}
$$

where the factors $\hbar$ and $g$ are absorbed in the normalization factor of $\xi_{s}$. The reader should also note that a second order term of $\chi_{s}$, which is $O\left(l_{h}^{4}\right)$ has been neglected in $j_{s}$, to be consistent with the neglect of $U_{q u}$. One can readily see that the definition of the 
norm (43) coincides in this approximation with the Klein-Gordon scalar product in the corresponding curved space.

The local coordinate transformation

$$
\widetilde{d t}=d t+\frac{v_{0}(x) d x}{s^{2}(x)-v_{0}^{2}(x)}, \quad d \widetilde{x}=d x
$$

proposed in Ref. 4 can be also written as

$$
d \widetilde{x}^{\mu}=\left(\begin{array}{c}
\tilde{d t} \\
d \widetilde{x}
\end{array}\right)=\Lambda_{\nu}^{\mu} d x^{\nu}
$$

It allows us to rewrite the density and the current in the form

$$
\begin{gathered}
\widetilde{\varrho}_{s}=-i \frac{s^{2}(\widetilde{x})}{s^{2}(\widetilde{x})-v_{0}^{2}(\widetilde{x})}\left[\left(\partial_{\tilde{t}} \xi_{s}^{*}\right) \xi_{s}-\xi_{s}^{*} \partial_{\tilde{t}} \xi_{s}\right], \\
\widetilde{j}_{s}=-i\left[v_{0}^{2}(\widetilde{x})-s^{2}(\widetilde{x})\right]\left[\left(\partial_{\widetilde{x}} \xi_{s}^{*}\right) \xi_{s}-\xi_{s}^{*} \partial_{\widetilde{x}} \xi_{s}\right],
\end{gathered}
$$

which have different signs in the subsonic $v(\widetilde{x})<s(\widetilde{x})$ and supersonic $v(\widetilde{x})>s(\widetilde{x})$ regions.

Now we use the density (47) in Eq. (43) in order to calculate the norm. The corresponding integral is separated in two regions outside the "black hole" $\widetilde{x}<-l_{r}$ (left) and inside it $\widetilde{x}>l_{r}$ (right) (see Figure 1). We may neglect the contribution of the narrow region $|x|<l_{r}$ in the integral where full solution of the second Eq. (13), rather than Eq. (44), should be used. As a result the norm takes the form

$$
<\vartheta_{s}, \vartheta_{s}>\approx \int_{-\infty}^{-l_{r}} d \widetilde{x} \widetilde{\varrho}_{s}+\int_{+l_{r}}^{\infty} d \widetilde{x} \widetilde{\varrho}_{s}=<\vartheta_{s}, \vartheta_{s}>_{\text {left }}+<\vartheta_{s}, \vartheta_{s}>_{\text {right }}
$$

The function $\widetilde{\varrho}_{s}$ in Eq. (47) diverges at $|\widetilde{x}| \rightarrow 0$, which could have resulted in the divergent contribution of the neglected region of integration. However, it is important to emphasize that Eq. (477) holds only outside the narrow region $|\widetilde{x}|>l_{r}$ near the Mach horizon. Within this region, $|\widetilde{x}|<l_{r}$, we have to return to Eq. (43). Although infinities are indicated as the integration limits in Eq. (48), the principal contributions come from the regions $\min \{1 / \alpha, 1 / \nu\}>|x|>l_{r}$. Since both $\chi_{s}$ and $\xi_{s}$ are regular at $|x|<l_{r}$ we will get only a small correction to the norm (47) from this narrow region, which can be neglected as long as $l_{h} \ll \min \{1 / \alpha, 1 / \nu\}$.

The integrals on the two sides of the horizon (left and right) approximately obey the relation

$$
<\vartheta_{s}, \vartheta_{s}>_{\text {left }}=-e^{2 \pi \operatorname{Im} \gamma}<\vartheta_{s}, \vartheta_{s}>_{\text {right }}
$$


due to the analytical properties of the function $\xi_{s}$. The total negative norm (48) is cut into the left moving positive frequency state and the right moving negative frequency state. The left state propagates against the flow "outside the black hole". Its relative weight is

$$
N(\omega)=\left(e^{2 \pi \operatorname{Im} \gamma}-1\right)^{-1}
$$

where

$$
\operatorname{Im} \gamma=\frac{2 \omega}{3 \bar{s} \alpha}+\frac{1}{162} \frac{\omega^{3} l_{r}^{3}}{\bar{s}^{3}}
$$

contains a correction due to the QP proportional to the third power of the frequency and, hence, $N(\omega)$ deviates from the Planck distribution for the black body radiation. This result can be also understood as a black body radiation spectrum with the frequency dependent temperature

$$
T_{H}(\omega)=\frac{T_{H}(0)}{1+\frac{1}{108} \frac{\omega^{2} l_{h}^{2}}{\bar{s}^{2}}}
$$

For not very high frequencies $\omega \ll \bar{s} / l_{r}$ this dependence may be neglected and $N(\omega)$ becomes the standard Planck distribution with the effective Hawking temperature

$$
T_{H}(0)=\frac{3 \hbar \bar{s} \alpha}{4 \pi k_{B}}
$$

\section{CONCLUSIONS}

The paper analyzes the role of the QP in the behavior of straddled fluctuations in the nearest vicinity of the Mach horizon. These are fluctuations attempting to propagate against the transonic flow that rips them into two parts, one of which propagates against the subsonic flow outside the "black hole", whereas the other part is taken away by the supersonic flow and "falls down" inside the black hole. The fluctuations are characterized by the singular behavior (28) in the region $l_{r} \ll x \ll \min \{\bar{s} / \omega, 1 / \alpha\}$, which on the quantum level leads to the Hawking radiation from the horizon. The singularity at the horizon $|x| \rightarrow 0$ is regularized due to the QP at the scale $x<l_{r}$ (40). This scale $l_{r}$ is usually larger than the healing length $l_{h}$, which plays in this system the role of the Planck length. Consequently it is the length $l_{r}$ which is relevant for the regularization rather than the "Planck length" $l_{h}$ (healing length). This regularization length can also be deduced from Eq. (57) of Ref. 31. However the author did not make it explicit. The regularization removes the divergency in the norm (48) and makes the procedure meaningful. The 
influence of the QP can be felt also outside this scale at $|x|>l_{r}$ since the exponent $\gamma$ in Eq.(39), contains now corrections due to the QP. They result in a deviation of the high frequency tail of the spectrum of Hawking radiation from the Planck black body radiation distribution.

Finally, we want to mention that neglecting QP results in a scale invariance of the equation of motion for $|k| \rightarrow \infty$. This is the origin of the occurrence of the singular solution $\chi_{s} \propto x^{\gamma-1}$ at $|x| \rightarrow 0$. The regularization we have found close to the horizon after accounting for QP is based on breaking this scale invariance for $|k| \rightarrow \infty$ (see Appendix)

Acknowledgment. We are grateful to L. Frankfurt, N. Pavloff, M. Reuter, M. Schwartz, G. Shlyapnikov and A. Soffer for helpful discussions. VF is indebted to J. Gutenberg University, Mainz, where he stayed during sabbatical, for hospitality. Support of United States - Israel Binational Science Foundation, Grant N 2006242 is acknowledged. RS also gratefully acknowledges financial support by MPI-PKS, Dresden.

\section{Appendix A: Function $\chi$ at $|x| \rightarrow 0$}

We try to elucidate here the mathematical features leading to the singularity at the horizon and its regularization. Without attempting mathematical rigor we present a qualitative discussion. Let us start with the case where the QP is neglected. For a stationary solution with variation in the $x$ direction only, we get from Eq. (32)

$$
\partial_{k} \ln \chi_{k}=\frac{\nu^{2}-2 \nu k}{i \alpha k(2 \nu-3 k-i \alpha)}
$$

for $l_{h}=0$ or for $|k| \rightarrow \infty$

$$
\partial_{k} \ln \chi_{k} \approx-\gamma_{0} \frac{1}{k}
$$

If $\chi(x)$ is a generalized homogeneous function, i.e.

$$
\chi(\widetilde{x})=\lambda^{\gamma} \chi(x), \quad \widetilde{x}=\lambda x
$$

for all real scaling parameters $\lambda$, then its Fourier transform fulfills the condition

$$
\chi_{\widetilde{k}}=\lambda^{(\gamma+1)} \chi_{k}, \quad \widetilde{k}=\lambda^{-1} k .
$$


Making use of (A2) and $\partial_{\widetilde{k}}=\lambda \partial_{k}$ it is easy to prove that

$$
\partial_{\widetilde{k}} \ln \chi_{\widetilde{k}} \approx-\gamma_{0} \frac{1}{\widetilde{k}}
$$

i.e Eq. (A1) is scale invariant. Taking the QP into account Eq. (32) results in

$$
\partial_{k} \ln \chi_{k} \sim \frac{i}{6} l_{r}\left(l_{r} k\right)^{2}
$$

from which it follows that

$$
\partial_{\widetilde{k}} \ln \chi_{\widetilde{k}} \sim \frac{1}{\lambda^{3}} \frac{i}{6} l_{r}\left(l_{r} k\right)^{2}
$$

i.e. Eq. (A33) is not scale invariant. This is consistent with the fact that its r.h.s., which involves the dimensionless quantity $\left(l_{r} k\right)^{2}$, explicitly depends on the length scale $l_{r}$. The latter has been found to be the scale below which regularization occurs. Taking in the expansion of $v(x)$ and $s(x)$ (close to the horizon) higher order terms in $x$ into account higher order derivatives with respect to $k$ will occur in Eqs. (30). This will prevent the decoupling of the fields $\xi_{k}$ and $\chi_{k}$ and therefore will hamper the analytical calculation of $\xi(x, t)$ and $\chi(x, t)$. Nevertheless the breaking of scale invariance still holds and is generic. Consequently, the regularization close to the horizon should be robust.

1 S. W. Hawking, Commun. Math. Phys., 43, 199 (1975).

2 S. W. Hawking, Phys. Rev., D 13 , 191 (1976).

3 N. D. Birrel and P. C. W. Davies "Quantum fields in curved spaces", Cambridge University Press, Cambridge, 1984

4 W. G. Unruh, Phys. Rev. Lett., 46, 1351 (1981).

5 T. A. Jacobson and G.E. Volovik, Phys. Rev. D 58, 064021 (1998).

6 B. Reznik, Phys. Rev. D, 62, 044044 (2000).

7 S. Giovanazzi, Phys.Rev.Lett., 94, 061302 (2005).

8 C. Barcelo, S. Liberati, and M. Visser, Phys. Rev. A, 68, 053613 (2003).

9 I. Carusotto, S. Fagnocchi, A. Recati, R. Balbinot, and A. Fabbri, New J. Phys., 10, 103001 (2008).

10 A. Recati, N. Pavloff and I. Carusotto, Phys. Rev., A 80, 043603 (2009).

11 P. D. Nation, M. P. Blencowe, A. J. Rimberg, and E. Buks, Phys. Rev. Lett., 103, 087004 (2009). 
12 I. Fouxon, O.V. Farberovich, S. Bar-Ad and V. Fleurov, Europhys.Lett., 92, 14002 (2010).

13 T. G. Philbin, C. Kuklewicz, S. Robertson, S. Hill, F. König, and U. Leonhardt, Science, 319, 1367 (2008).

14 O. Lahav, A. Itah, A. Blumkin, C. Gordon, S. Rinott, A. Zayats, J. Steinhauer, Phys. Rev. Lett. 105, 240401 (2010).

15 F. Belgiorno, S. L. Cacciatori, M. Clerici, V. Gorini, G. Ortenzi, L. Rizzi, E. Rubino, V. G. Sala, and D. Faccio, Phys.Rev.Lett., 105, 203901 (2010).

16 G. Rousseaux, C. Mathis, P. Maissa, T. G. Philbin, and U. Leonhardt, New J. Phys., 10, 053015 (2008).

17 G. Rousseaux, P. Maissa, C. Mathis, P. Coulet, T. G. Philbin, and U. Leonhardt, New J. Phys., 12, 095018 (2010).

18 S. Weinfurtner, E. W. Tedford, M. C. J. Penrice, W. G. Unruh, G. A. Lawrence, Phys. Rev. Lett., 106, 021302 (2011).

19 L. I. Sedov, Two Dimensional Problems in Hydrodynamics and Aerodynamics, Interscience Publ., New York, 1965.

20 L. D. Landau and E. M. Lifshits, Fluid Mechanics, Pergamon Press, Oxford, 1987.

21 E. Madelung, Z. Phys., 40, 322 (1927).

22 J. H. Marburger, Progr. Quant. Electr. 4, 35 (1975).

23 T. Jacobson, Phys. Rev. D 44, 1731 (1991).

24 T. Jacobson, Phys. Rev.D 48, 728 (1993).

25 W. G. Unruh, Phys. Rev. D 51, 2827 (1995).

26 S. Corley, Phys. Rev. D 55, 6155 (1997).

27 J. Macher and R. Parentani, Phys. Rev. D 79, 124008 (2009).

28 J. Macher and R. Parentani, Phys. Rev. A 80, 043601 (2009).

29 R. Brout, S. Massar, R. Parentani, and Ph. Spindel, Phys. Rev. D 52, 4559 (1995).

30 S. Corley and T. Jacobson, Phys. Rev. D 54, 1568 (1996).

31 S. Corley, Phys. Rev. D 57, 6280 (1998).

32 S. Corley and T. Jacobson, Phys. Rev. D 59, 124011 (1999).

33 U. Leonhardt, T. Kiss, and P. Öhberg, Journal of Optics B: Quantum and Semiclass. Opt. 5, S42, (2003).

34 U. Leonhardt, T. Kiss, and P. Öhberg, Phys. Rev. A 67, 033602 (2003). 
35 W. G. Unruh and R. Schützhold, Phys. Rev. D 71, 024028 (2005).

36 R. Schützhold and W. G. Unruh, Phys. Rev. D 78, 041504 (R) (2008).

37 S. Finazzi and R. Parentani, Phys. Rev. D 83, 084010 (2011).

38 C. Barcelo, S. Liberati, and M. Visser, Living Rev. Relativ. 8, 12 (2005).

39 A. L. Fetter und J. D. Valecka, Quantum theory of many-particle systems, McGraw-Hill, (1971).

40 G. P. Agrawal, Nonlinear Fiber Optics (Academic Press, New York) 1995.

41 N. Bleistein and R. A. Handelsman, Asymptotic Expansions of Integrals, Dover Punl., New York (1986).

42 T. Damour, and R. Ruffini, Phys. Rev. D 14, 332 (1976).

43 T. Damour and M. Lilley, arXiv:0802.4169v1 (2008) 